\title{
Integration of pulsed-laser ESPI with spatial domain modal analysis
}

\author{
H. Van der Auweraer ${ }^{\mathrm{a}, *}$, H. Steinbichler ${ }^{\mathrm{b}}$, C. Haberstok ${ }^{\mathrm{c}}$, R. Freymann ${ }^{\mathrm{c}}$ and D. Storer ${ }^{\mathrm{d}}$ \\ ${ }^{a}$ LMS International, Interleuvenlaan 68, B-3001 Leuven, Belgium \\ ${ }^{\mathrm{b}}$ Labor dr. Steinbichler, Neubeuern, Germany \\ ${ }^{\mathrm{c}}$ BMW AG, München, Germany \\ ${ }^{\mathrm{d}}$ Centro Ricerche Fiat, Orbassano (TO), Italy
}

\begin{abstract}
The present paper discusses the various critical elements of a modal testing system based on pulsed-laser holographic ESPI measurements. Such system allows making very high spatial resolution measurements on panel-like structures at frequencies that are of relevance for the vibro-acoustic behavior.

Next to the optical parts, the integration with the modal analysis procedure, including the integration of geometry and response information, is reviewed. The ESPI based Image-domain frequency response functions are accumulated frequency by frequency. Special attention is paid to the modal parameter extraction of the large DOF number data, for which a special data reduction scheme was developed. The approach has been applied to several case studies related to car panels. The research was conducted in the context of the Brite/Euram project SALOME and the EUREKA project HOLOMODAL.
\end{abstract}

\section{Introduction}

Experimental modal analysis (EMA) is a wellestablished approach to analyze and model the dynamic behavior of mechanical structures. The upper frequency limit where EMA can be applied is depending on the type of the structure: car chassis, suspension, engine block, aircraft wing, etc. The limiting factors with increasing frequency are:

- The increasing spatial complexity of the modes, requiring a more dense grid of response degrees of freedom.

- The increasing modal density and modal overlap requiring an adequate frequency resolution in the FRF measurements.

Especially for light-weight, panel-type structures, the weight of the acceleration sensors starts to influence the structural response. Non-contact measurement techniques have been proposed to address this problem.

*Corresponding author. Tel.: +32 16384 200; Fax: +32 16384 350; E-mail: herman.vanderauweraer@1ms.be.
The most widely spread approach is the one based on a laser-Doppler vibrometer, which can be equipped with a computer controlled position/orientation system. All DOF's of interest are "scanned" in a sequential way, with the laser-vibrometer acting for each location as a single-point velocity response transducer. Such scanning laser devices are currently available and their control and data processing is fully integrated with commercial modal test and analysis systems.

A second approach to non-contact measurements is based on holography. The main advantage of holography techniques is that they allow whole-field measurements of a vibrating structure at one specific time instance. The recent evolution in the field of ESPI (Electronic Speckle Pattern Interferometry), allowing to use direct CCD-camera imaging of vibration states at specific, controlled, time instances, has renewed the interest in this approach. At first, ESPI holography was merely used to just qualitatively "image" the vibration patterns, for example at resonance frequencies. Recent research projects however, have extended this approach into a tool for deriving quantitative data, in the form of whole-field Frequency Response Function (FRF) maps. This was done first for Stroboscopic CW (continuous 


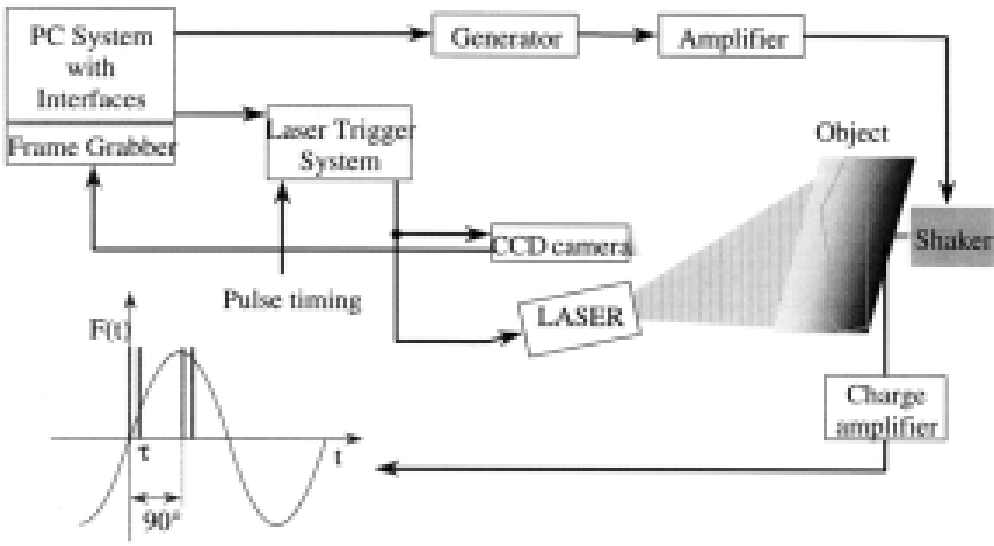

Fig. 1. Pulsed-laser ESPI set-up.

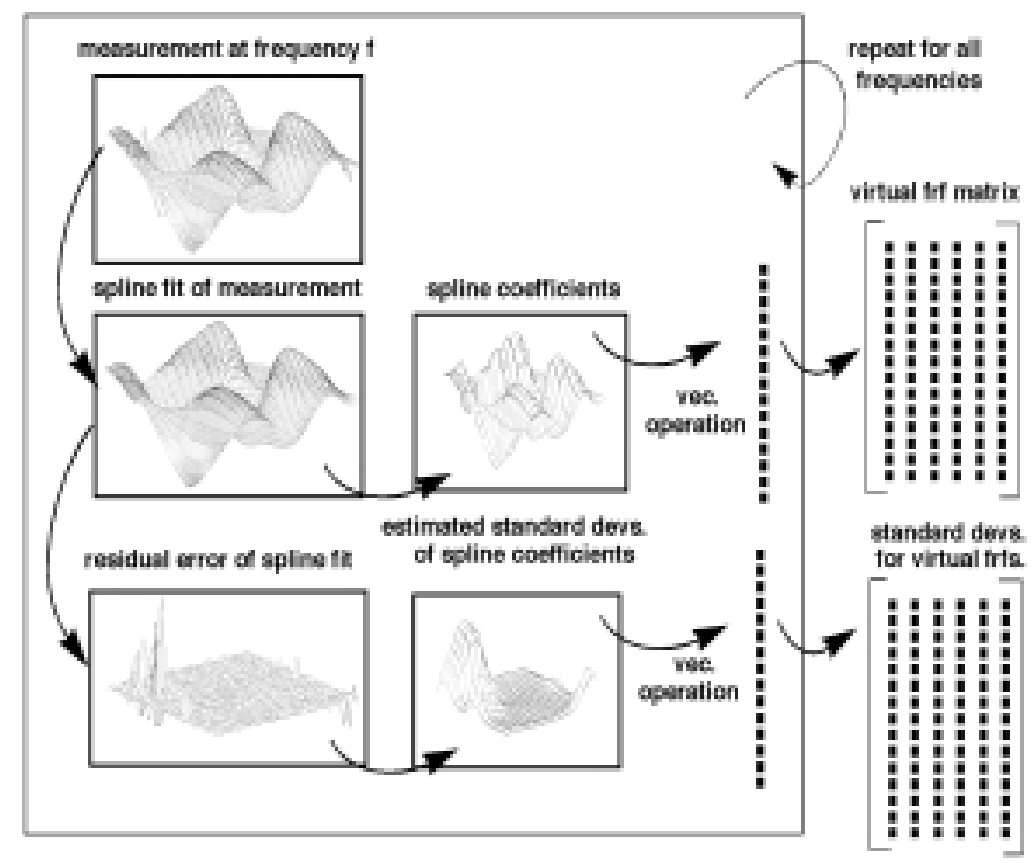

Fig. 2. Data reduction procedure.

wave) laser illumination, and, very recently, also for pulsed laser illumination. These data can then be processed by modal parameter estimation methods or they can directly be used as an FRF model for applications such as acoustic radiation prediction or FE updating.

\section{ESPI-holography based FRF measurements}

The principles of Electronic Speckle Pattern Interferometry are well documented $[1,2]$. For the purposes of quantitative vibration analysis, a specific procedure is used. First, specklegrams are obtained for well-defined deformation states, for example using stroboscopic CW illumination, or pulsed lasers, triggered to the excitation force. The optical phase is extracted using timestepping $[3,4]$ or based on spatial phase differences [5].

When the phase fields for two deformation states are known (e.g. one in rest, one a vibration snapshot or alternatively two vibration instances), they can be subtracted, resulting in contrast-rich fringe patterns. Since the values of the thus derived relative phase range 


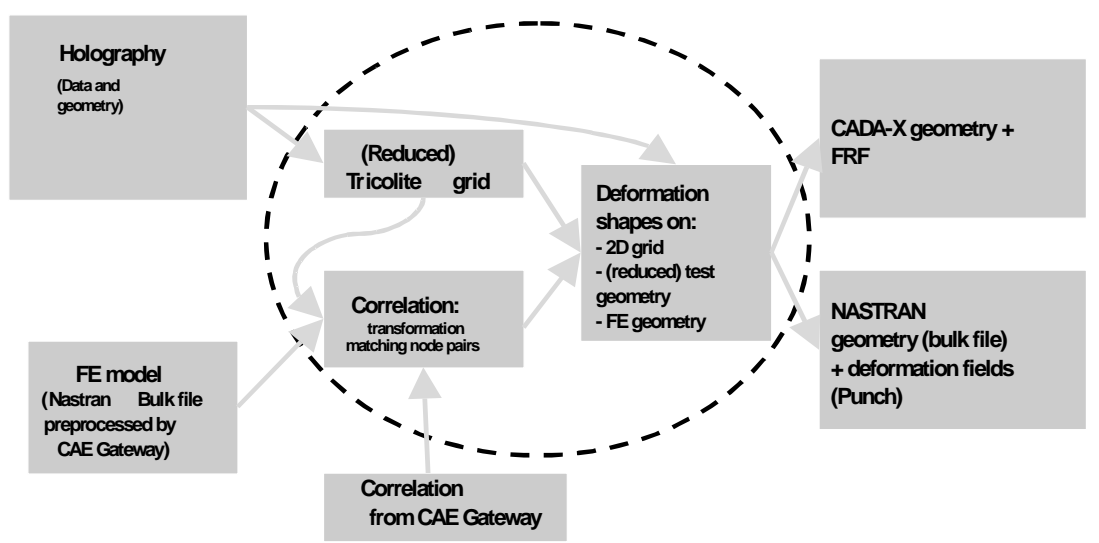

Fig. 3. HoloModal CAE Integration.
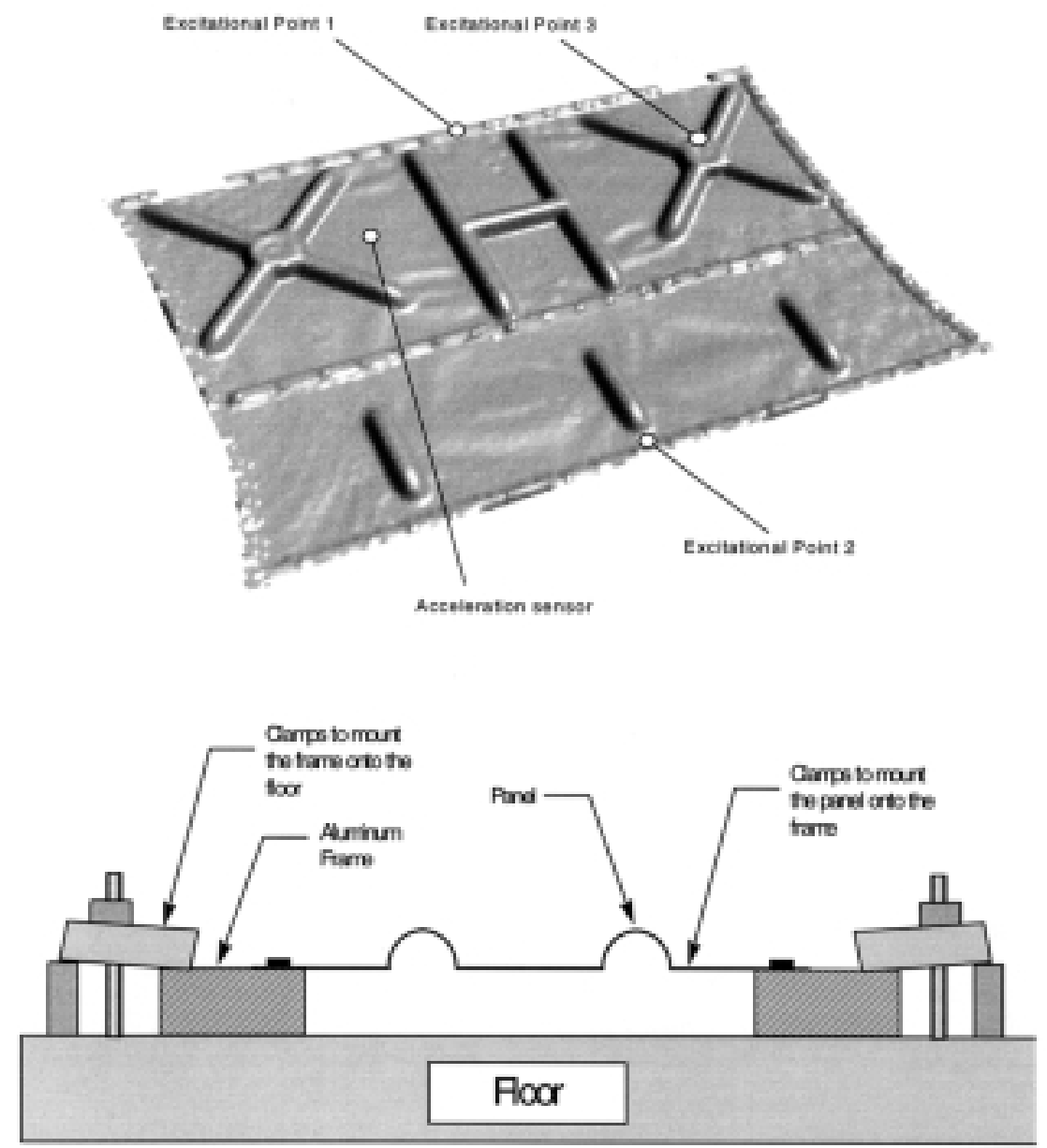

Fig. 4. Panel construction

between 0 and $2 \pi$, the phase has to be unwrapped before it can be scaled to relative displacement. This requires an important amount of advanced signal processing.
Adequate and "intelligent" algorithms for automatic image filtering and phase unwrapping are the key to automating the complete measurement procedure. 


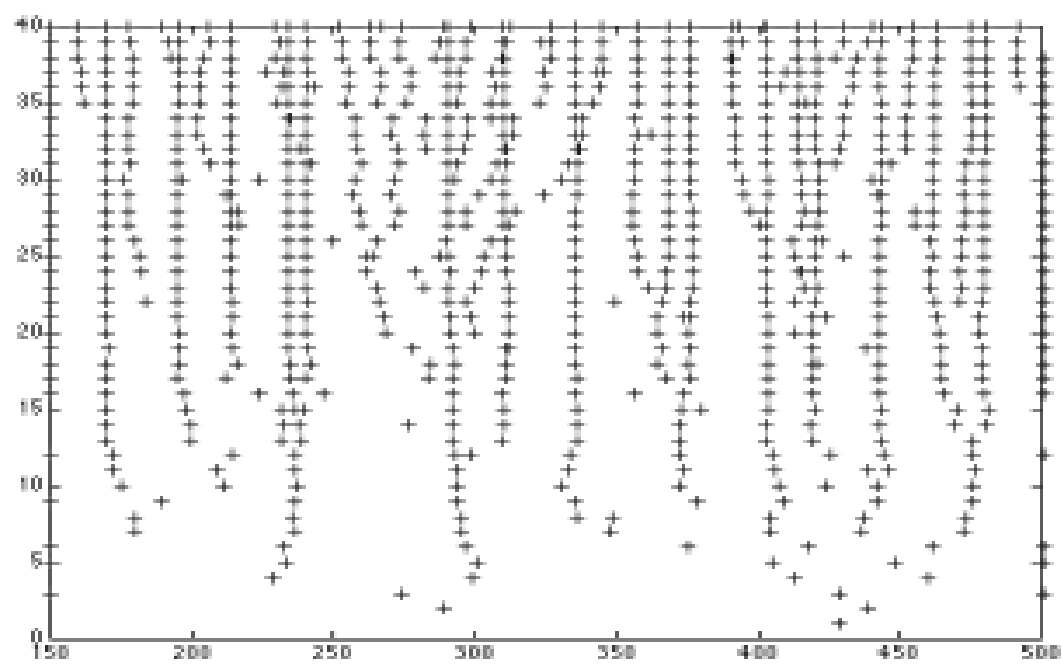

Fig. 5. Stabilization diagram LSCE.

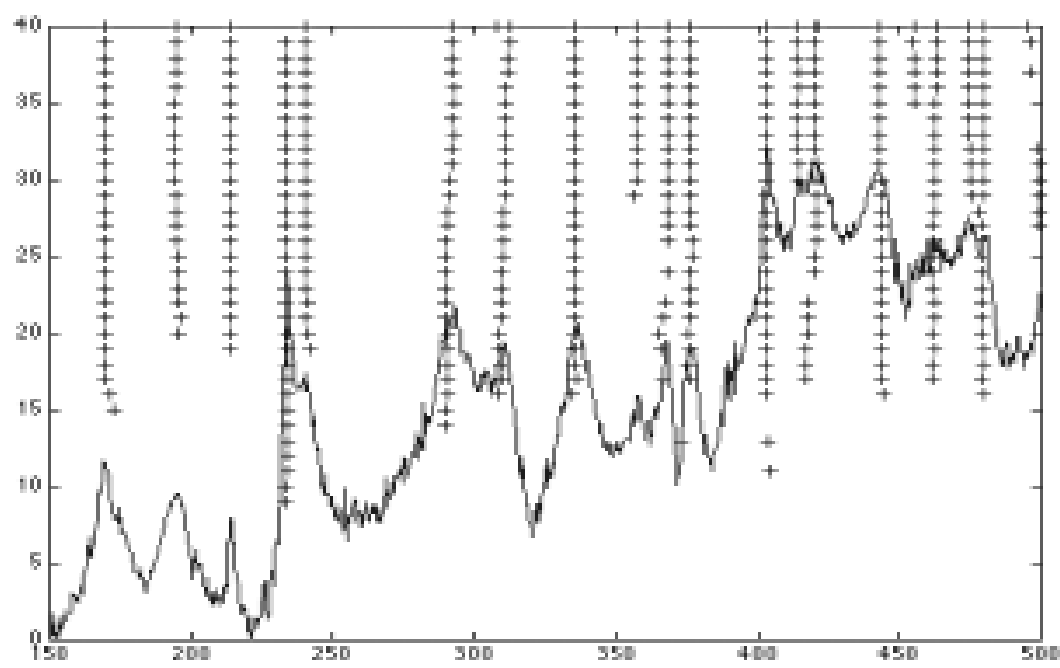

Fig. 6. Stabilization diagram MLE.

In the classical ESPI approach, the vibration measurement is performed at frequencies of maximal vibration. The vibration responses are then used as estimates of the mode shapes [6,7]. In the present project, the goal of the measurements was to obtain complexvalued vibration amplitudes (in principle at pixel resolution) that, in reference to the excitation force, yield an "image" of frequency response functions (FRFs). These FRF images are accumulated over a range of frequencies $[8,9]$.

The stepped-sine excitation technique is used hereto. To determine the unknown value of the complex amplitude, at least two measurements per frequency need to be made at (at least) two different time points [6]. When more than two time points are used, the complex amplitude is calculated by a least squares procedure, which not only reduces the effects of noise in the measurement data, but also has a filtering effect of harmonic 
disturbances in the excitation and/or response signals.

In practice, a hybrid approach using a preliminary broadband test with a few accelerometers, combined with a fine-resolution stepped sine sweep around the frequencies of high dynamic response, turned out to be very effective, combining the speed of the direct response approach with the accuracy of an FRF-based modal parameter estimation.

In the HOLOMODAL and SALOME projects, two set-ups were realized, one using a stroboscopic CW laser and one using a double pulse laser. The complete set-up of laser, optics, camera and automated phase unwrapping and image processing was developed by Labor Dr. Steinbichler (Fig. 1). For acquiring the double-pulse images, a special measurement head has been developed. It allows the subsequent storage of 2 full frames of $1280 \mathrm{X} 1024$ pixels for a minimal pulse separation of $0.5 \mu \mathrm{s}$. This camera renders it possible to store the specklegram related to both pulses with a 12-bit resolution.

\section{Modal parameter estimation}

The identification of modal parameters from ESPIFRF measurements has to deal with the following requirements:

- Capability to deal with an extremely large number of degrees of freedom (in principle pixel resolution is available)

- Capability to treat high order systems (since the modal density increases with frequency)

- Capability to treat non-uniform FRF data (for example by increasing the frequency resolution near resonance).

Furthermore, the method has to be robust with respect to non-uniform data quality (e.g. due to poor reflecting areas), or unequal vibration levels (due to local modes). Preferably, it should assess and exploit the noise information in the data.

While "classical" modal parameter estimation methods, such as the Least-Squares Complex Exponential technique can still be used (e.g. by subsampling the image domain to a sizeable grid, and by restricting the measurements to equidistant excitation frequencies), they are by far not optimal in the sense of the requirements described above. A new approach has hence been developed, based on a data reduction procedure and a high-order frequency domain Maximum Likelihood parameter estimation algorithm.

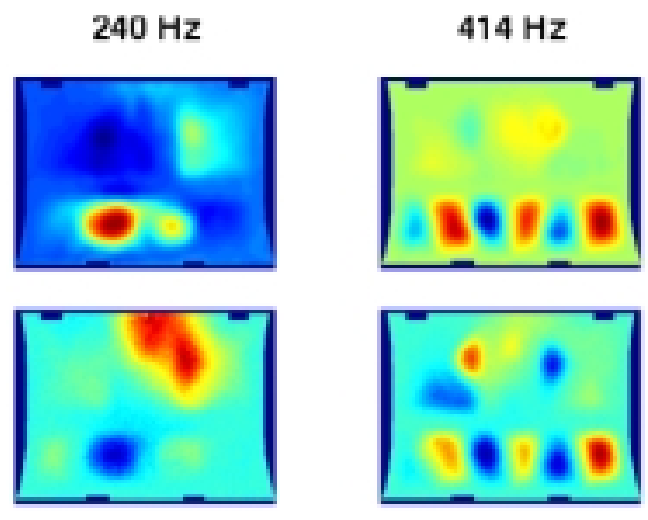

Fig. 7. Mode shapes (upper: MLE, lower: FRF response).

The data for estimating the modal parameters are FRFs with image resolution. Since the number of effective degrees of freedom will only be a small portion of the number of measured responses, a data reduction procedure can be used to compress the information and to reduce the parameter estimation time.

The most straightforward way is to subsample the image and to perform the parameter estimation on a sub-grid. However, this way, no use is made of the redundancy in the data. Several approaches for data condensation have been proposed. The selected approach is based on a two-dimensional spline regression for data reduction. It is discussed in detail in $[10,11]$. Since the transformation is a linear one, the coefficients can be used as "virtual measurements" for estimating the modal parameters (poles and "virtual" domain mode shapes). The estimated "virtual mode shapes" can then be converted back (or expanded) into the full grid of physical measurements.

The determination of the spline basis is done only once using the measurement near the highest resonance since complex operating shapes are occurring in the higher frequency range. The use of splines as regressive functions has important implications on the data reduction procedure. Firstly this increases the computational speed. But, even more importantly, since the basis of B-splines is a local one, noise information can be estimated from the spline fit assuming that the noise distribution is locally invariant. This procedure is elaborated in more detail in [11]. The result is that, next to the "virtual" FRF matrix, also a matrix with uncertainty information is available. Figure 2 summarizes the data reduction procedure.

Once the data are condensed into a sizable matrix of virtual FRFs, the actual parameter estimation can be performed. As the estimator has to be used to model 


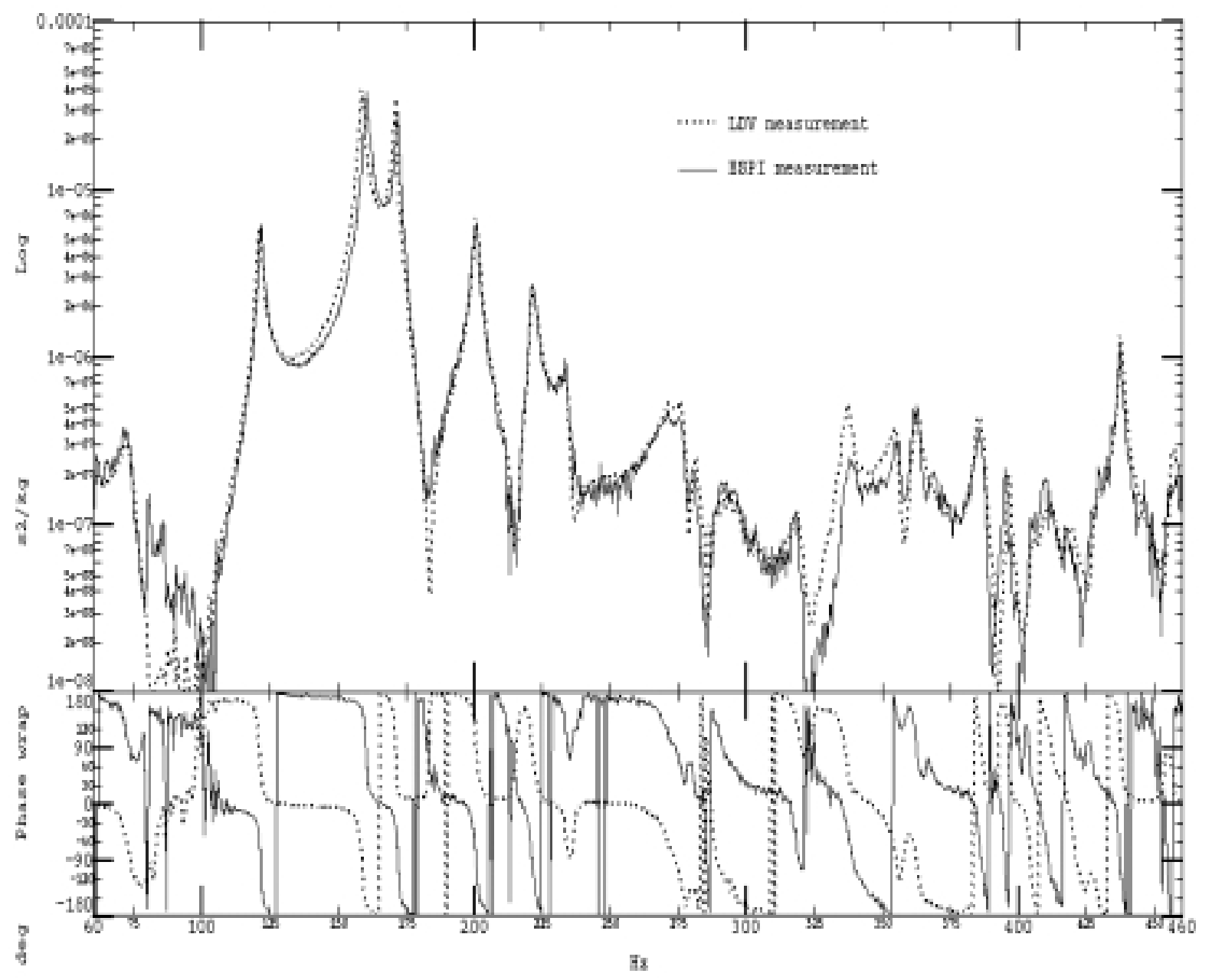

Fig. 8. FRF of a high response point measured with ESPI and LDV (dotted line).

systems in the medium frequency range, it should be able to handle high-order models. Also, because the recorded holographic images can be noisy, it is necessary to use an estimator that has a high accuracy. For this goal, an identification scheme is proposed, based on a weighted frequency domain Maximum Likelihood algorithm. The MLE implementation used is the fast and robust logarithmic version of the MLE algorithm elaborated in [12]. An essential element is that noise information (when available, for example from the data reduction scheme) is explicitly used as a weighting function in the definition of the cost function. This dramatically improves and facilitates the parameter estimation process. Also, the resulting parameter estimates are complemented by a set of corresponding uncertainties.

\section{Geometry and cae integration}

Important to the use of the ESPI results is their integration with a geometry reference. The measurement of the object geometry uses an LCD fringe projector (TRICOLITE system) projecting different gratings on the object. The same camera as used for the ESPI views the illuminated object. The co-ordinates of the geometry are then calculated by triangulation, using the light code and the pixel position on the CCD camera. Each pixel of the ESPI image is given a co-ordinate.

The availability of a geometry information also enables the use of the test data for correlation with FEM data and/or for use of the test data as boundary conditions in a FEM/BEM forced response calculation. For, in many cases, the main purpose of the use of laser 


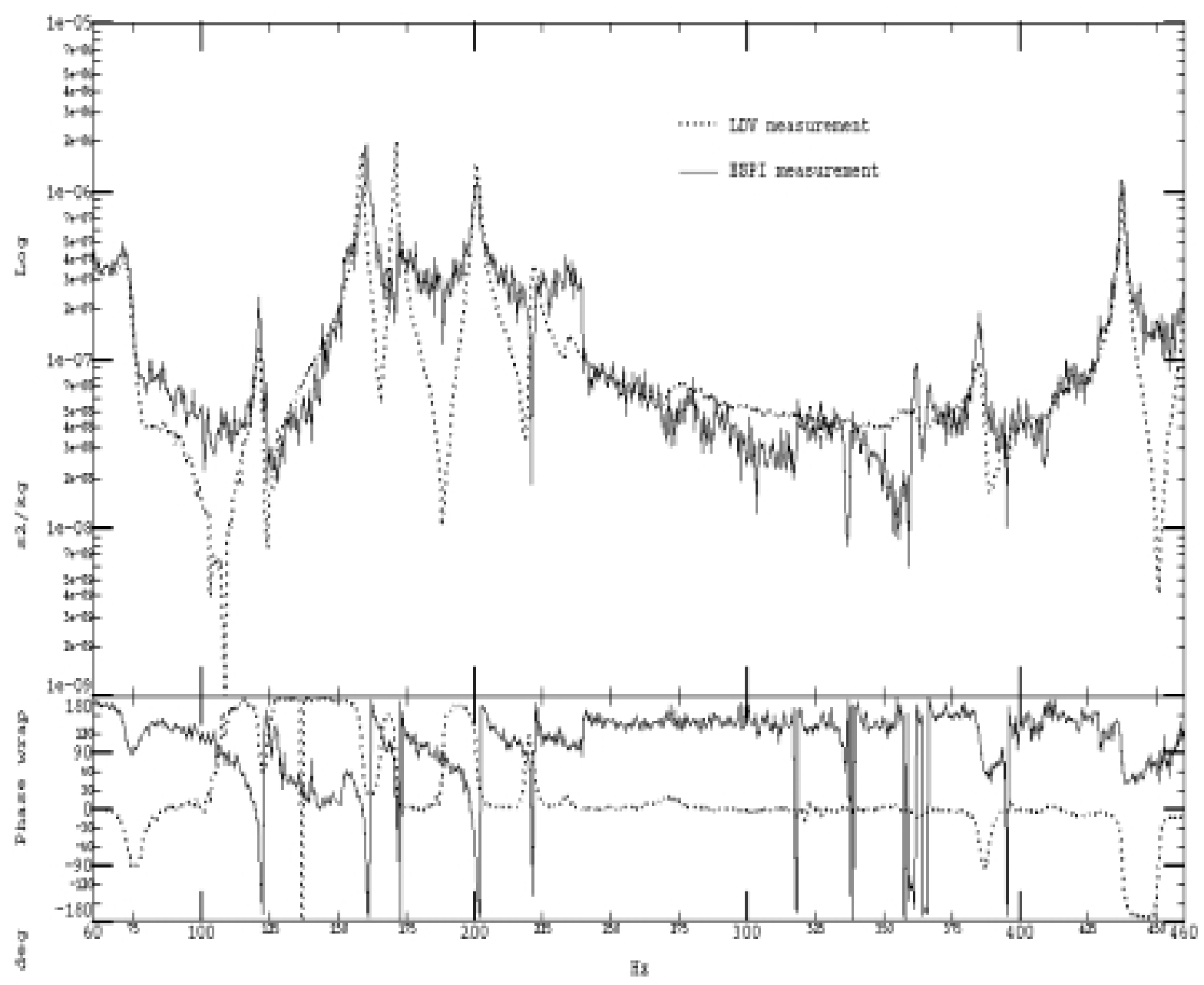

Fig. 9. FRF of a low response point measured with ESPI and LDV (dotted line).

holography is the validation of panel FE models. Typically, such FEM models contain a large number of "nodes" where the information is available. In general, experimental modal analysis models are defined for a small number of geometrical points. For the case of Holographic modal analysis results however, the situation can be the inverse.

Different ways of correlating the test and numerical models exist. On one hand, all points of the holographic image can be correlated to interpolation functions of the FE model. This uses all ESPI information, but it requires access to the FE interpolation functions, which is a problem for commercial FE software. As the image processing corresponds to a data reduction, it is unnecessary to transfer all data. Therefor, a preselection of the available holographic results by defining which of these points correspond to the FE model is supported. An overview of the related data entities is given in Fig. 3.

Once the experimental holographic data are available in a CAE reference frame (projected on an FE raster), the data can be used in a many CAE applications such as correlation, validation and updating of FE models. In the same way the measured FRF data can be used as boundary conditions to vibro-acoustic prediction codes, for example in acoustic BE or FE codes.

\section{Application to single panels}

The ESPI-HOLOMODAL approach was first validated on a ribbed panel, representative for typical au- 


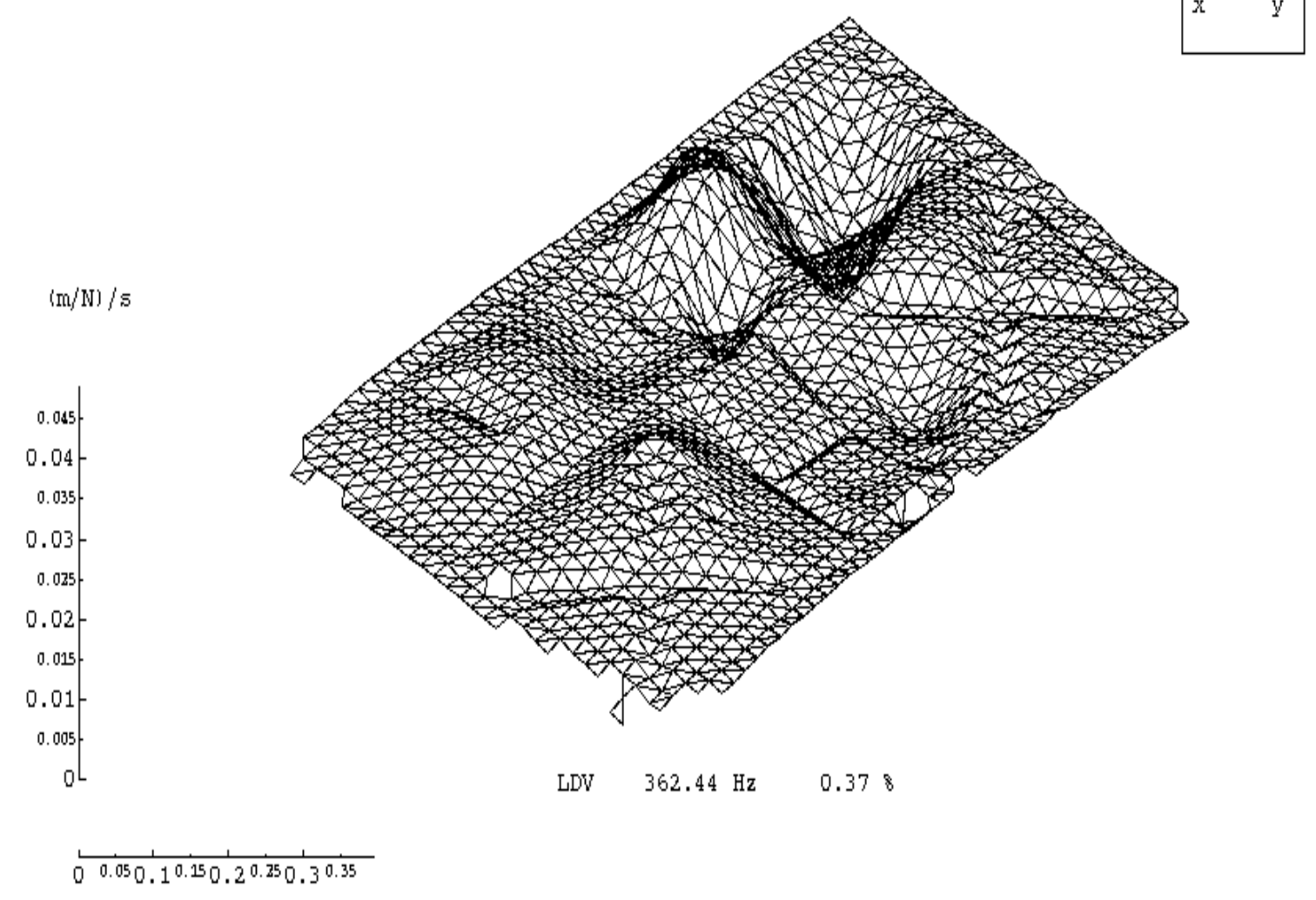

Fig. 10. Mode at $362.44 \mathrm{~Hz}$ (LDV).

tomotive panels such as a cabin or trunk floor plate or a door panel.

\subsection{Test of a panel mounted on a rigid frame}

In the first test set-up, the ribbed panel was mounted on a rigid aluminum frame, which was clamped rigidly to the floor (Fig. 4). An acceleration sensor was glued onto the panel to compare its measurements with the ones from holography.

A first series of test was performed using the CWESPI system. Stepped sine excitation (with 16 time steps per frequency) was performed between 150 and $500 \mathrm{~Hz}$ in steps of $0.5 \mathrm{~Hz}$. From these data, the modal model identification was performed, comparing the LSCE and MLE methods. A mesh of 1700 points was generated through subsampling of the images. The stabilization diagrams for both methods with the MLE using the noise information are shown in Figs 5 (LSCE) and 6 (MLE). They clearly indicate the drastically improved stabilization behavior of the MLE.

Some representative mode shapes are shown in Fig. 7 ( 240 and $414 \mathrm{~Hz}$ ). Both methods are compared and also the FRF value at these frequencies (which would be taken as the mode shape in the classical resonance response analysis approach) is given. The direct response at $240 \mathrm{~Hz}$ clearly shows a residue of the excitation; the $414 \mathrm{~Hz}$ response does not uncouple the mode completely from the neighboring $403 \mathrm{~Hz}$ one.

\subsection{ESPI-LVD comparison}

Another validation test was performed comparing the ESPI system with a scanning laser-Doppler vibrometer (LDV). To correlate the panel with a previously created FE model, several reference points were chosen. Dur- 


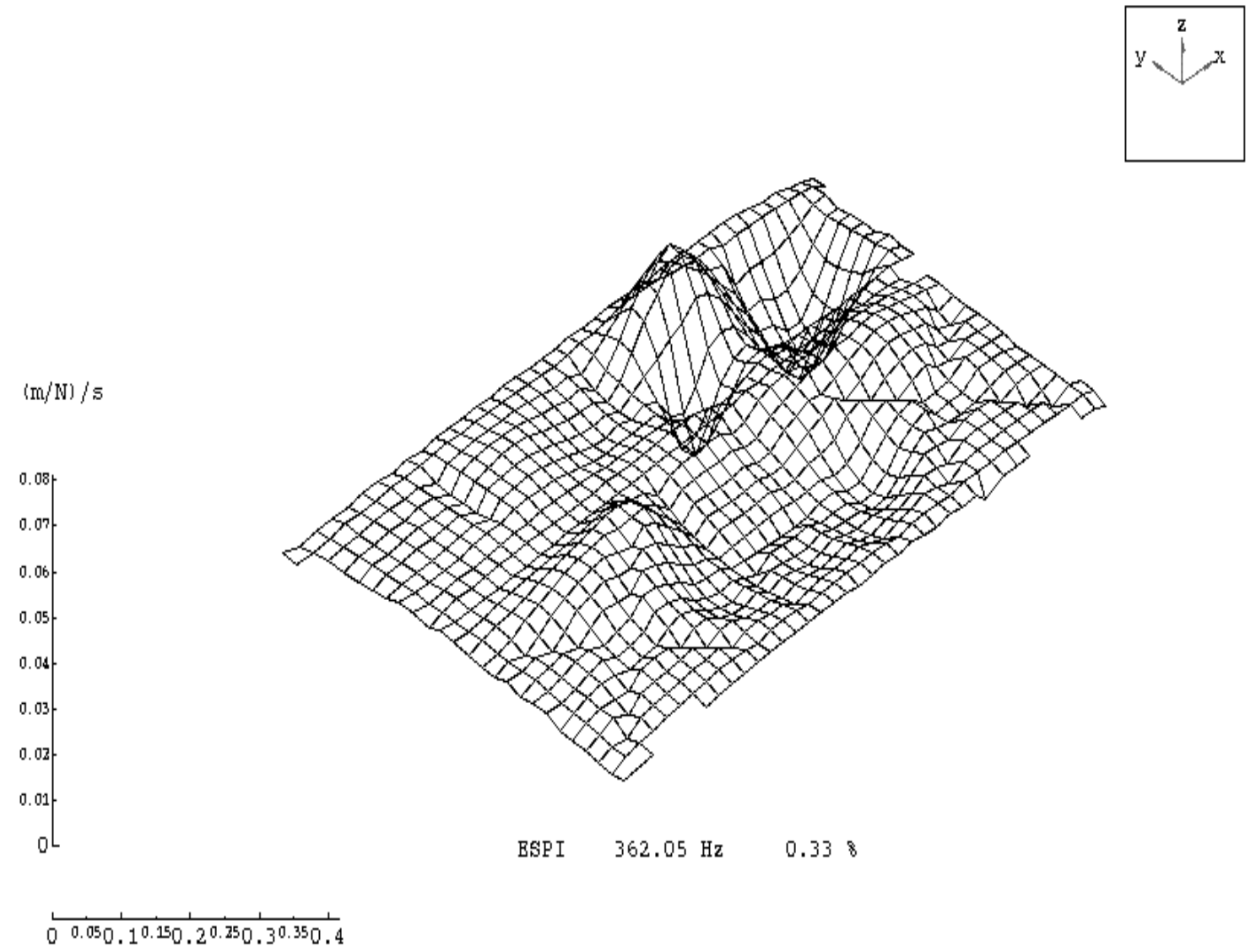

Fig. 11. Mode at $362.05 \mathrm{~Hz}$ (ESPI).

ing the actual LDV test, the laser beam was guided to every point of the FE model, and the FRF from 0 to $800 \mathrm{~Hz}$ was measured at 1432 points.

The ESPI measurement head was mounted above the test panel. The TRICOLITE system was used to measure the Geometry of the panel and to get a correlation of the measured geometry to the camera pixels. The ESPI test was executed from 60 to $460 \mathrm{~Hz}$ using a frequency step of $0,5 \mathrm{~Hz}$. After applying a grid, the number of points was reduced to 934 .

The frequency response functions, measured with the LDV and the ESPI system are compared for the same measurement points. In Figs 8 and 9, the FRFs are shown for a point with a high response and for a point with a low response.

It can be seen from these figures that the ESPI measurements are noisier, especially for the low response point. The amplitude is good, but there are differences in the phase (in addition to a $180^{\circ}$ shift). It should be noted that these are raw data, without any spatial smoothing.

A modal analysis was then performed on both data sets between 60 and $470 \mathrm{~Hz}$. In both cases the stabilization is very good. The same modes are found back in both data sets. For some modes there is a little frequency shift, probably due to a little change of the measurement set-up between the two tests (some weeks apart). The damping estimates are the same for both analyses. The mode shapes are very much the same, as can be seen in Figs 10 and 11 for 1 mode. The differences in the generalized modal parameters (unity maximum scaling) are somewhat larger.

Overall, it can be concluded that these validations proved the inherent capabilities of the system. In view of data quality, the use of multiple sampling steps per frequency is advised. In view of the measurement time, the laser cooling and recharge time are the dominant ones. 


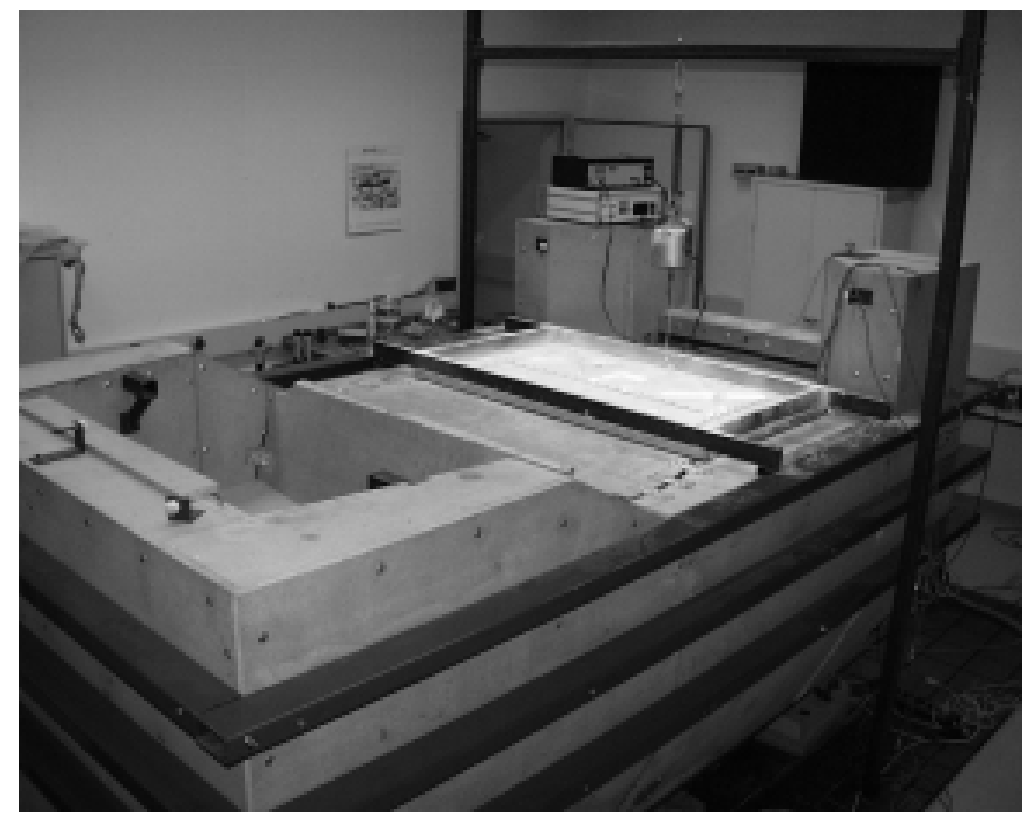

Fig. 12. Photograph of the measurement setup in the laboratory.

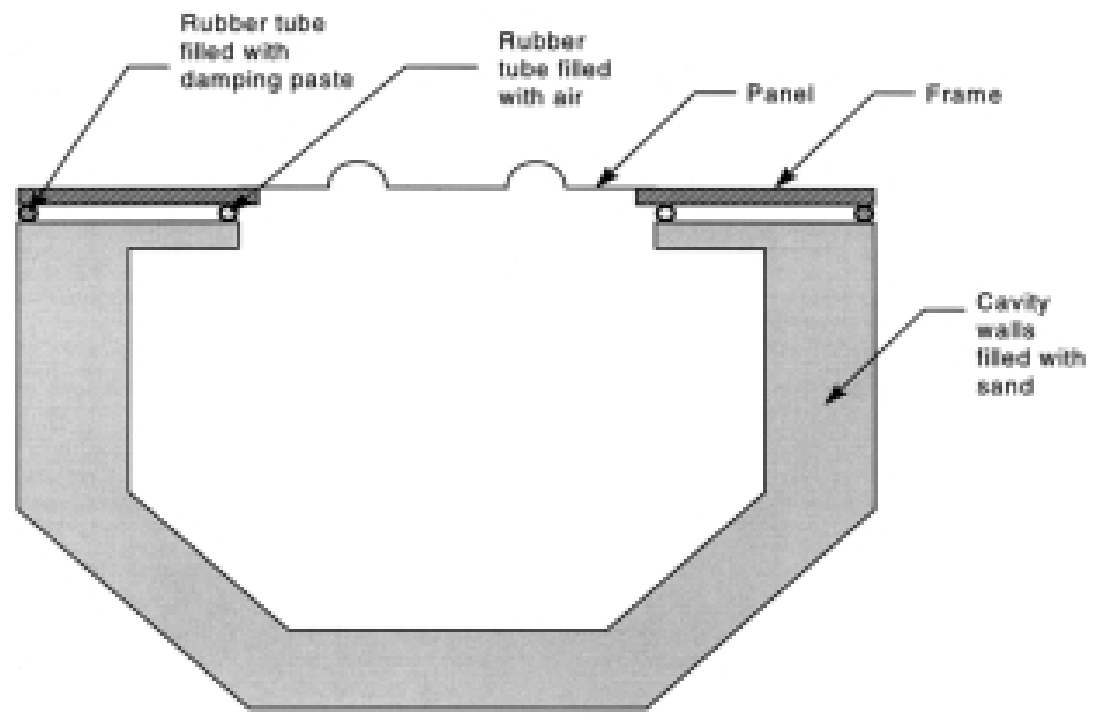

Fig. 13. Bearing and sealing of the frame.

\subsection{Test of panel mounted to a frame structure and coupled to a cavity with rigid walls}

In another test, the panel attached to a frame structure was mounted on top of a rigid walled cavity (Figs 12 and 13). Holographic measurements were then performed using the completely automated Pulsed laser ESPI system. Stepped sinusoidal excitation was ap- plied in the range $50-561 \mathrm{~Hz}$ in steps of $1 \mathrm{~Hz}$. Two time steps per frequency were used.

The acquisition time needed for one phase image was $1 \mathrm{~min}$. This time is mainly dependent on the laser cooling. The ruby laser is specified to 1 double pulse per minute because it gets too hot when emitting more pulses. The recharging of the laser only takes about 12 secs, as well the processing of one phase image.

Based on these data, a first modal analysis was per- 


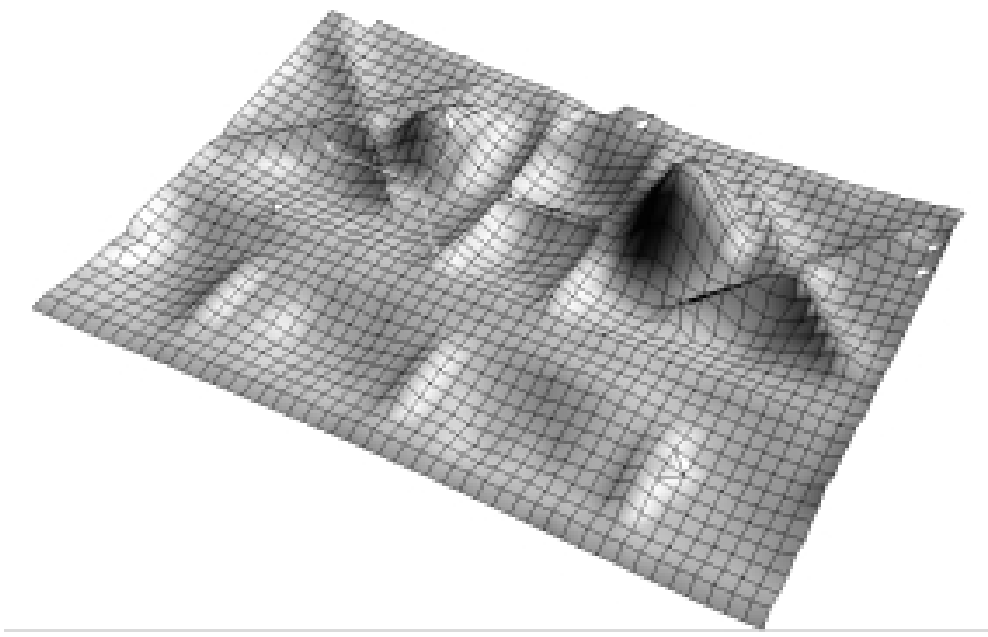

Fig. 14. Mode shape at $444 \mathrm{~Hz}$.

Table 1

Modal parameter results

\begin{tabular}{ccc}
\hline Pole & Frequency $(\mathrm{Hz})$ & Damping $(\%)$ \\
\hline 1 & 121.619 & 8.5 \\
2 & 151.493 & 3.5 \\
3 & 176.834 & 2.8 \\
4 & 209.042 & 0.7 \\
5 & 218.422 & 0.9 \\
6 & 240.679 & 1.7 \\
7 & 253.417 & 1.3 \\
8 & 275.111 & 1.2 \\
9 & 321.678 & 1.3 \\
10 & 361.812 & 0.5 \\
11 & 406.479 & 0.8 \\
12 & 418.606 & 0.6 \\
13 & 444.732 & 0.7 \\
14 & 467.627 & 0.5 \\
15 & 492.222 & 0.7 \\
16 & 500.111 & 0.5 \\
17 & 520.537 & 0.6 \\
18 & 50.000 & \\
19 & 561.000 & \\
20 & & \\
\hline
\end{tabular}

formed using the MLE method. Table 1 shows the pole values. A sample mode shape at $444 \mathrm{~Hz}$ is shown in Fig. 14.

The FRF (displacement/force) data, acquired by the ESPI system, were transformed into velocity boundary conditions for an acoustic boundary element model (BE model) of the cavity (Fig. 15). The BE model was created using 5 elements per acoustic wavelength for the highest frequency to be calculated Since the walls of the cavity were covered with damping material, a representative frequency dependent impedance value was applied to the boundaries of the BE model. Also, FRF measurements were made using a pressure sensor inside the cavity with respect to the force applied to the vibrating panel. They were compared with the calculated pressure due to the measured boundary conditions (Fig. 16).

\section{Tests on a vehicle}

In the last discussed case, a complete, partially trimmed, vehicle was analyzed with respect to panel induced interior noise. In a first step, a panel contribution analysis was performed to identify the most critical panels. The tests were executed on a chassis dynamometer. These tests are further discussed in [13].

In general it was observed that for the partiallytrimmed vehicle under investigation, the main contribution to the internal sound pressure level is accounted for by the roof and floor panels over the $75-165 \mathrm{~Hz}$ frequency range, whereas the windscreen has relevant contributions particularly over the $50-70 \mathrm{~Hz}$ and $135-$ $165 \mathrm{~Hz}$ ranges.

In a second step, a detailed dynamic analysis of the windscreen was performed using the ESPI system. Stepped sine shaker excitation $(40-160 \mathrm{~Hz})$ was applied hereto at the front engine mount. Figure 17 shows some of the operational deflection shapes of the windscreen at different frequencies: $50 \mathrm{~Hz}$ (near the resonance frequency identified by the previous tests), $90 \mathrm{~Hz}, 120 \mathrm{~Hz}$ and $160 \mathrm{~Hz}$ (where the windshield also had a significant contribution).

These results show the importance to first execute a panel contribution analysis to identify the panels responsible for the interior noise. The detailed ESPI analysis can then be limited to those critical panels. 


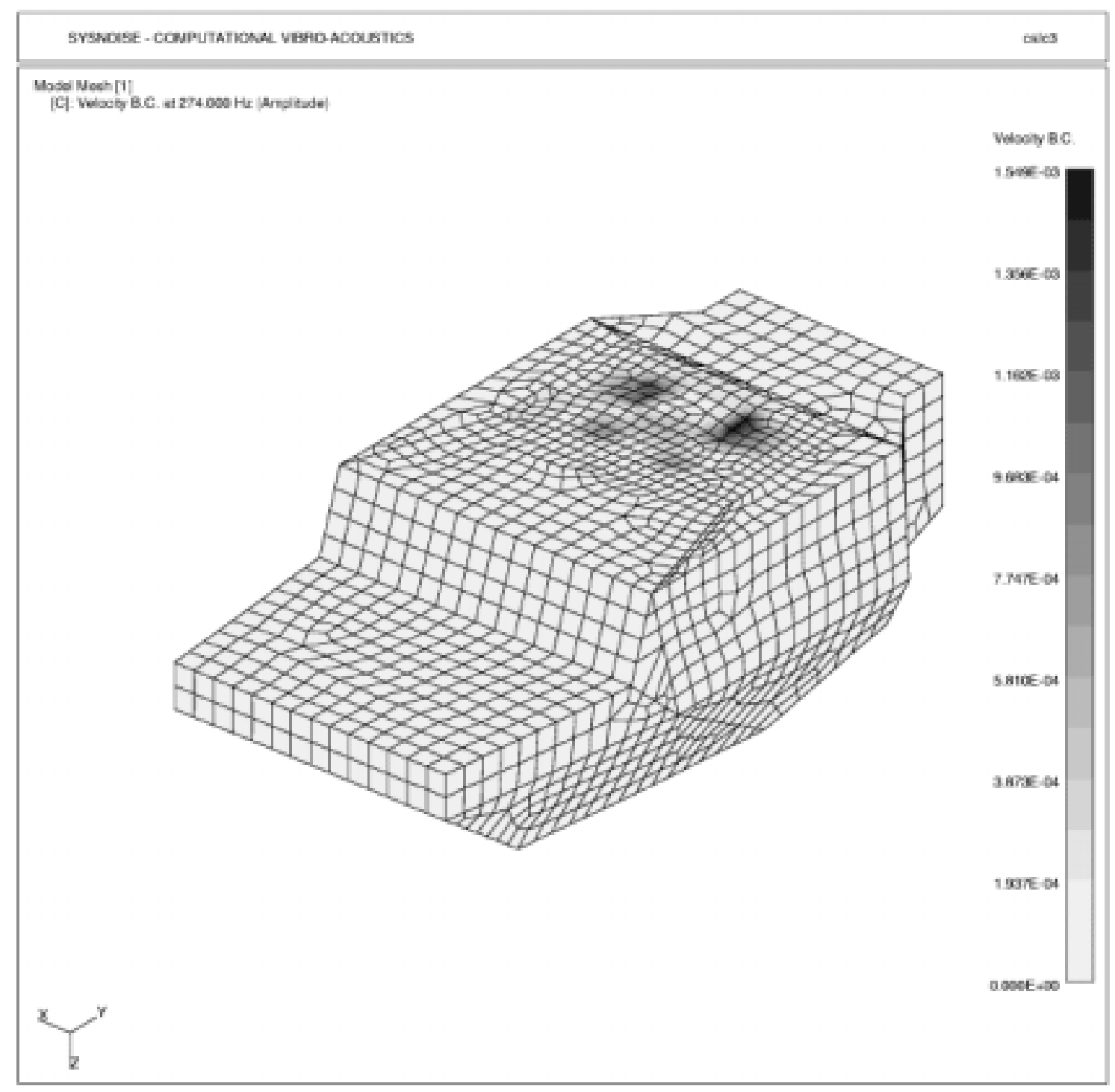

Fig. 15. BE model with panel boundary condition.

\section{Conclusion}

ESPI holography (both using stroboscopic CW and double pulse laser illumination) has evolved to a stage of industrial usability. Direct CCD imaging, rugged hardware design and automated image processing are key elements to this.

By integrating the ESPI vibration imaging with the stepped sine modal testing procedure, yielding ultrahigh resolution frequency response function data sets, the method has become fully compatible with the wellproven phase separation modal test and analysis procedure.
High performance spatial domain parameter estimation methods based on spline condensation and Maximum Likelihood parameter extraction allow to efficiently process the large volumes of data, and at the same time optimally take the available noise information into account. The method has been validated on various car-panel structures in an industrial test environment.

\section{Acknowledgements}

The presented research work was carried out in the framework of EUREKA project no. 1503, HOLO- 


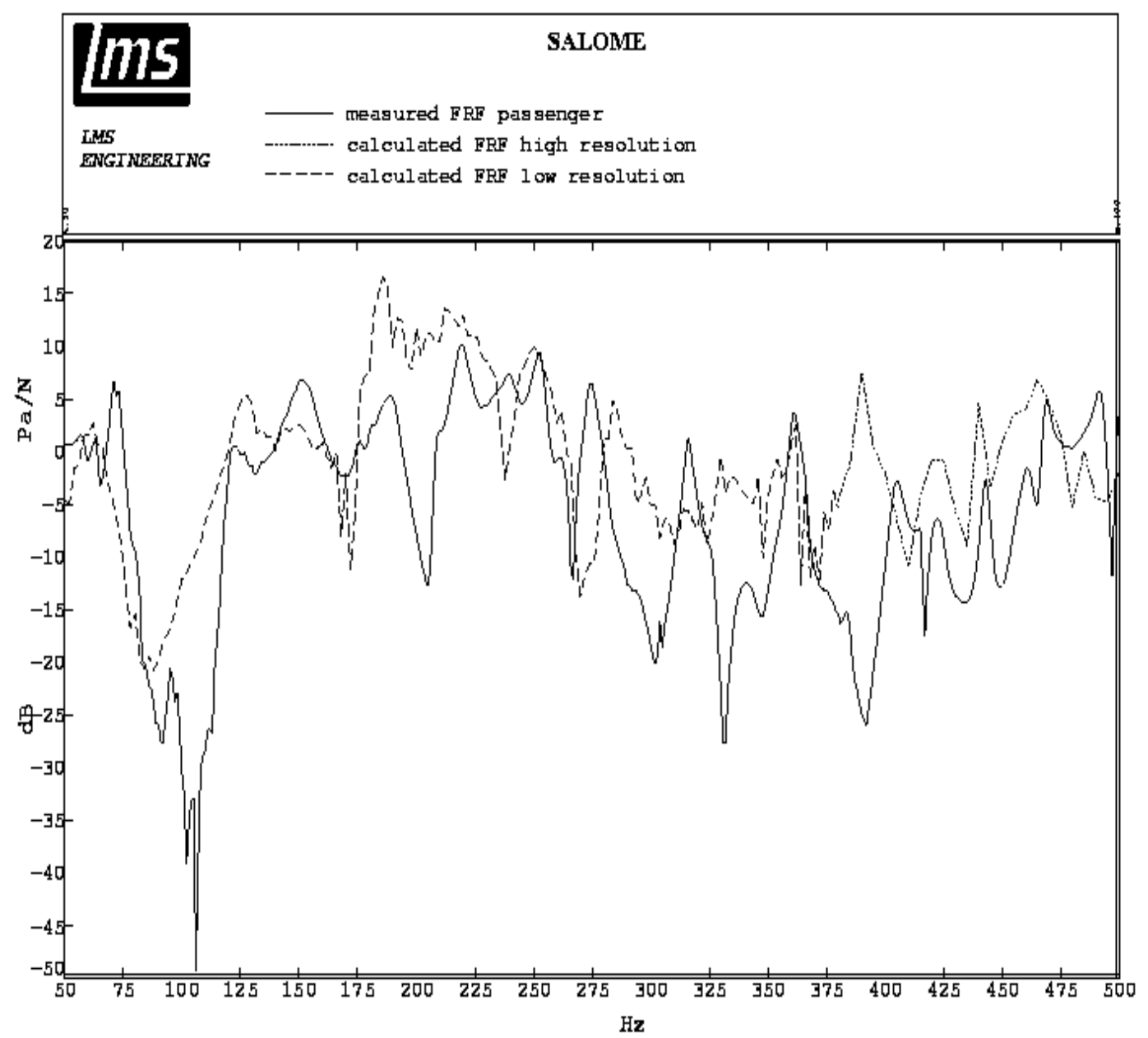

Fig. 16. Calculated versus measured pressure with respect to unity force at the panel.

MODAL and the Brite-Euram project SALOME. Partners in HOLOMODAL are: LMS International NV, BMW AG, Labor Dr. Steinbichler and the Vrije Universiteit Brussel. Partners in SALOME are the HOLOMODAL team together with K.U.Leuven, PSA and CRF. The project is supported by the Flemish Institute for the promotion of scientific and technological research in industry (IWT) and the European Community (EC).

\section{References}

[1] N.A. Halliwell, Optical methods for vibration measurement and visualisation, Proceedings of the International symposium on prediction of the noise emitted by vibrating structures, Senlis (F), March 26-28, 1991, pp. 221-241.

[2] G.A. Cloud, Optical methods of engineering analysis, Cambridge University Press.
[3] K. Creath, Phase-shifting speckle interferometry, Appl. Opt. 24 (1985), 3053-3058.

[4] R. Dändliker and P. Jacquot, Holographic interferometry and speckle methods, in: Optical Sensors, Sensors Vol. 5, VCH Verlagsgesellschaft, Weinheim (D), pp. 589-628.

[5] H. Steinbichler, Patent 0419936 B1, Verfahren und Vorrichtung zur Phasemessung von Strahlung, insbesondere Lichtstrahlung.

[6] V. Linet, X. Bohineust, F. Dupuy and C. Stawicki, Experimental modal analysis of structures using three-dimensional holographic interferometry: development and applications, Proceedings of the 17th international seminar on modal analysis, Leuven (B), September 23-25, 1992, pp. 1655-1676.

[7] P. Slangen, L. Berwart, C. de Veuster, J.-C. Golinval and Y. Lion, Digital speckle interferometry (DSPI): A fast procedure to detect and measure vibration mode shapes, Proceedings of the SPIE international conference on vibration measurements by laser techniques, Ancona (I), SPIE volume 2358, October 3-5, 1994, pp. 102-110.

[8] H. Klingele and H. Steinbichler, Holographic modal analysis for the separation of narrow-spaced eigenmodes, Proceedings of the SPIE international conference on vibration measure- 

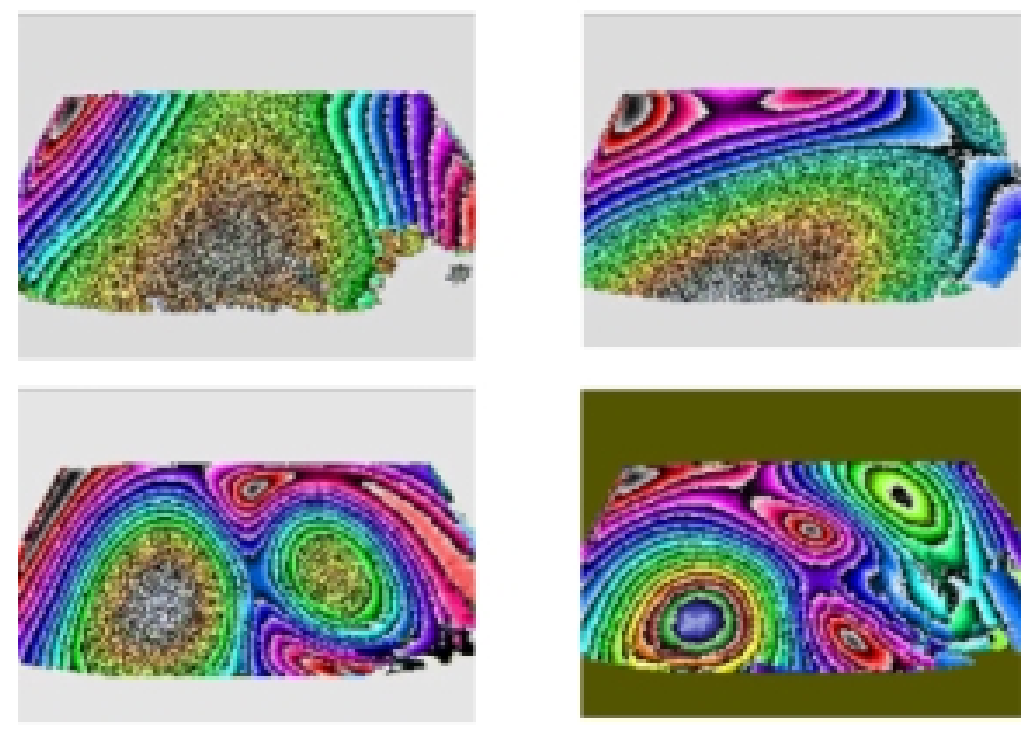

Fig. 17. Operational deflection shape amplitudes at $50 \mathrm{~Hz}$ (upper left), $90 \mathrm{~Hz}$ (upper right), $120 \mathrm{~Hz}$ (lower left) and $160 \mathrm{~Hz}$ (lower right).

ments by laser techniques, Ancona (I), SPIE volume 2868, September 23-25, 1996, pp. 412-417.

[9] C. Haberstok, R. Freymann and W. Honsberg, Holographic modal analysis with high spatial resolution, Proceedings of ISMA 23, Leuven (B), September 16-18, 1998, pp. 991-998.

[10] S. Vanlanduit, P. Guillaume and J. Schoukens, High resolution spatial domain modal parameter estimation of complex structures, Proceedings of the 16th international modal analysis conference, 1998, pp. 1629-1655.

[11] S. Vanlanduit, P. Guillaume and J. Schoukens, Development of a data reduction procedure with noise extraction for high res- olution optical measurements, Proceedings of the SPIE 3411 (1998), 357-365.

[12] P. Guillaume, P. Verboven and S. Vanlanduit, Frequencydomain maximum likelihood identification of modal parameters with confidence intervals, Proceedings of ISMA 23, Leuven (B), September 16-18, 1998, pp. 359-366.

[13] M. Vanolo, D. Storer and H. Van der Auweraer, Associating the vibrations of car body panels to the internal acoustic response, Proc. ISMA-25, Leuven (B), Sept. 13-15, 2000, pp. 13691376. 

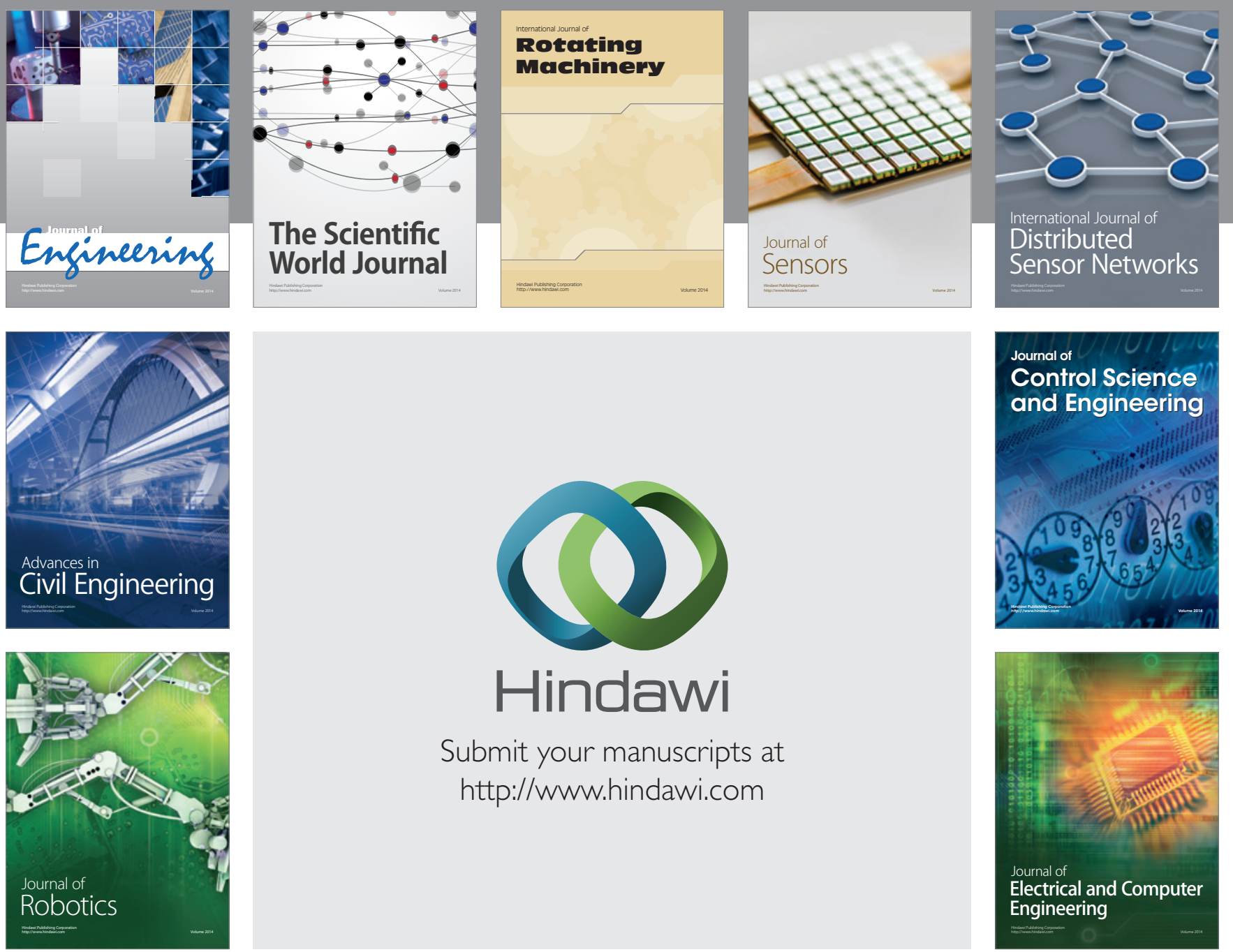

Submit your manuscripts at

http://www.hindawi.com
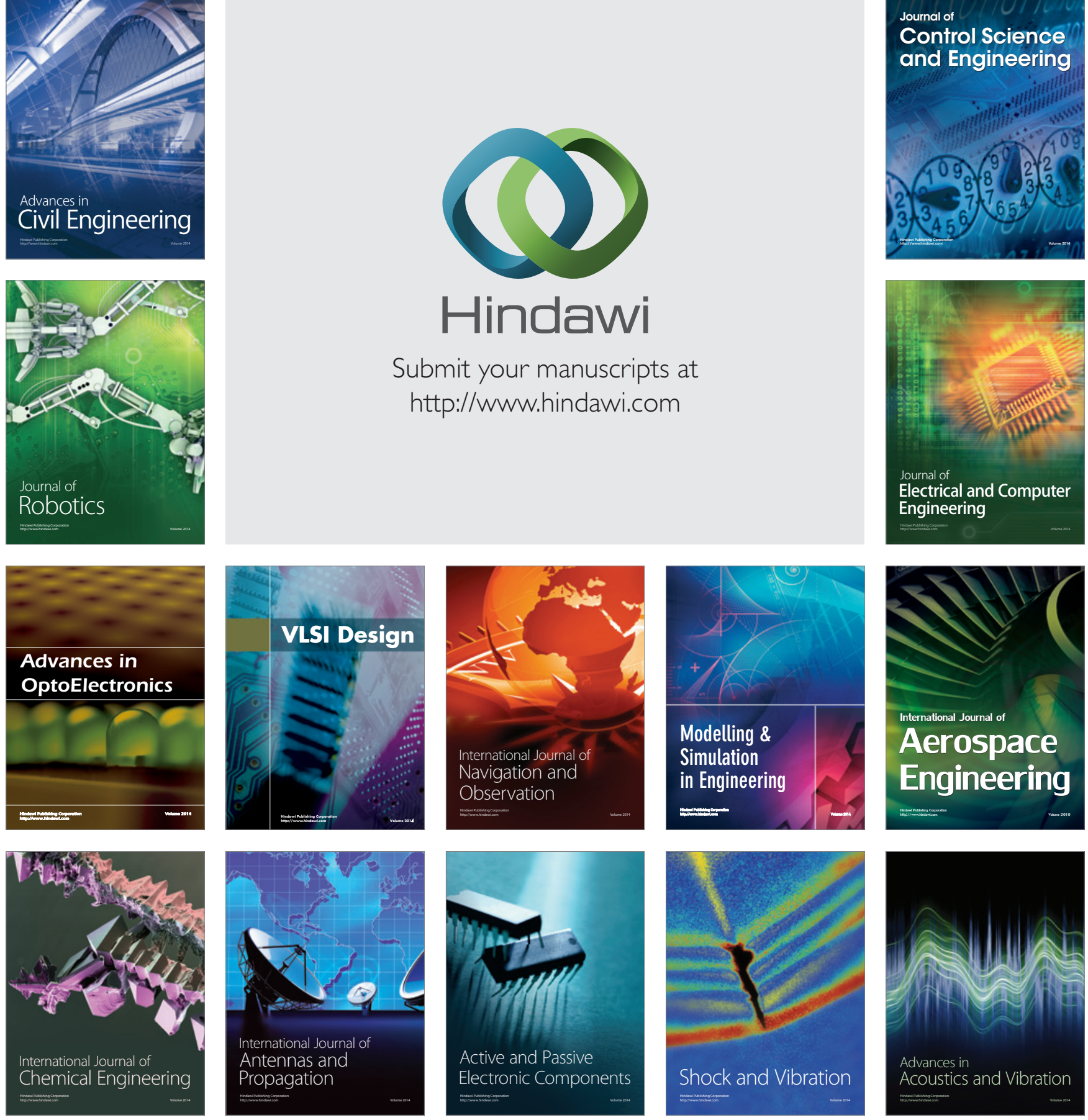